Revista peruana de biología 26(4): 521 - 524 (2019) doi: http://dx.doi.org/10.15381/rpb.v26i4.XXXX ISSN-L 1561-0837; elSSN: 1727-9933

Universidad Nacional Mayor de San Marcos

\section{Primer reporte de albinismo para el cuy silvestre Cavia tschudii (Mammalia: Rodentia)}

\author{
First albinism report for the wild guinea pig Cavia tschudii \\ (Mammalia: Rodentia)
}

$\begin{array}{ll}\text { Presentado: } & 20 / 09 / 2019 \\ \text { Aceptado: } & 08 / 11 / 2019 \\ \text { Publicado online: } & 16 / 12 / 2019\end{array}$

Correspondencia:

* Autor para correspondencia

Dámaso W. Ramirez: dramirezh@cientifica.edu.pe Manuel Quispe-López: manuel.artq@gmail.com Diego Marcelo-C: diego.marcelo@unmsm.edu.pe Victor Pacheco: vpachecot@unmsm.edu.pe

1 Panamericana Sur Km. 19 - Lima 42. Perú. 2 Departamento de Mastozoología, Av. Arenales 1256, Jesús María. Lima, Perú

3 Universidad Nacional Mayor de San Marcos, Museo de Historia Natural, Laboratorio de Florística, Av. Arenales 1256, Jesús María, Lima, Perú.

4 Santa Rita No 105, Dpto. 202, Urb. Huertos de San Antonio, Surco, Lima, Perú.

Otros datos de los autores / biografía:

Orcid Dámaso W. Ramirez: 0000-0003-4605-9422 Orcid Victor Pacheco: https:0000-0002-1005-135X

Citación:

Ramirez D.W, M. Quispe-López, D. Marcelo-Carranza y V. Pacheco. 2019. Primer reporte de albinismo para el cuy silvestre Cavia tschudii (Mammalia: Rodentia). Revista peruana de biología 26(4): 521 - 524 (Diciembre 2019). doi: http://dx.doi.org/10.15381/ rpb.v26i4.17218

Palabras clave: humedal costero; albinismo; mamíferos pequeños; patrones de coloración; fenotipos; desordenes cromáticos; Perú.

Keywords: Coastal wetland; albinism; coloring patterns; phenotypes; chromatic disorders; Peru; small mammals.

\section{Dámaso W. Ramirez*1,3, Manuel Quispe-López 2,4, Diego Marcelo- Carranza $^{2}$ y Víctor Pacheco ${ }^{2}$}

1 Dirección de Cursos Básicos, Universidad Científica del Sur, Lima, Perú. 2 Universidad Nacional Mayor de San Marcos, Museo de Historia Natural, Lima, Perú. 4 Centro de Ornitología y Biodiversidad (CORBIDI), Lima, Perú.

\section{Resumen}

En mamíferos silvestres los patrones de coloración atípica como el albinismo ocurren en baja frecuencia y en la literatura científica sus reportes son escasos. Por lo cual los hallazgos de este tipo son importantes para comprender mejor este fenómeno. En el presente trabajo realizamos el primer reporte de albinismo para el cuy silvestre Cavia tschudii (Rodentia) en la costa central de Perú. Este hallazgo también representa el primer registro de albinismo para el género Cavia en el Neotrópico.

\section{Abstract}

In wild mammals, atypical coloration patterns such as albinism occur at a low frequency and are poorly reported in the literature. Therefore, discovery of cases of coloration anomalies are important to better understand this phenomenon. Here, we describe the first reported case of albinism in Cavia tschudii "Wild Guinea Pig" from the central coast of Peru. This discovery also represents the first record of albinism for the genus Cavia in the Neotropics.

\section{Introducción}

Los patrones de coloración anormal en mamíferos son eventos raros en la naturaleza y escasamente reportados en la literatura (Robinson 1973, Acevedo \& Aguayo 2008, Acevedo et al. 2009, Romero \& Titira 2017, Romero et al. 2018). Estas coloraciones anormales son clasificados como albinismo, leucismo y melanismo, y ocurren debido a una ausencia o exceso del pigmento melanina en parte o todo el cuerpo (Berman et al. 2004, Acevedo \& Aguayo 2008, Acevedo et al. 2009, Stumpp et al. 2018). El albinismo o hipopigmentación es una anomalía genética que afecta la generación de melanosomas normalmente pigmentados dentro de melanocitos viables (Lamoreux et al. 2010), se manifiesta en condiciones recesiva de los alelos ocasionando una disminución drástica del color o la ausencia total en la piel, pelos y ojos, obteniendo como resultado individuos albinos con ojos rojos, piel blanca y un pelaje blanco o claro (Berman et al. 2004, Acevedo et al. 2009, Martinez-Coronel et al. 2013, Stumpp et al. 2018). Por otro lado, el leucismo se caracteriza por una pigmentación reducida en el pelaje, un blanco en la mayor parte del cuerpo o en parches, 
pero que todavía posee de forma normal el color de ojos y piel. Finalmente, el melanismo es el incremento en la cantidad de la pigmentación negra o oscura (Acevedo \& Aguayo 2008, Acevedo et al. 2009).

En mamíferos silvestres el albinismo es un evento inusual (Caro 2005, Romero et al. 2018), siendo reportado de forma rara en roedores (Romero et al. 2018), murciélagos (Treitler et al. 2013) y carnívoros (Blaszczyk et al. 2007). En el orden Rodentia, el taxón más diverso en mamíferos (2480 especies, Wilson et al. 2017), los casos de albinismos en poblaciones silvestres han ocurrido en menos del 2\% de sus especies (Romero et al. 2018). La baja frecuencia de albinismo en poblaciones silvestres podría ser un indicador de una selección negativa, debido a que su condición los haría fácilmente observables como depredador o presa, sumado a los efectos deletéreos de sus ojos (Martinez-Coronel et al. 2013). Así mismo, los estudios sobre la coloración y sus desordenes cromáticos han sido realizados principalmente en roedores domésticos como Mus musculus (ratón de laboratorio) (Beermann et al. 2004, Cieslak et al. 2011) y Cavia porcellus (cuy doméstico, "guinea pig"), ambas empleadas como modelo para evaluar coloraciones atípicas (Wright 1915, Dunn 1921, Robinson 1973, Bui \& Vingrys 1999).

Cavia tschudii Fitzinger 1857, pariente silvestre del cuy doméstico (C. porcellus), es un roedor nativo de los Andes de Sudamérica, con una distribución desde el norte de Chile, Argentina, Bolivia, hasta Perú, con un rango altitudinal desde el nivel del mar hasta los 4500 m (Dunnum 2015). En Sudamérica se tienen 8 subespecies de $C$. tschudii en las cuales se observan una coloración oscura del pelaje dorsal que pueden variar de color canela, marrón rojizo hasta un gris negruzco; mientras que el pelaje ventral tiene una coloración blancuzca a gris; dejando evidencia que hay una diferencia en las tonalidades del color del pelaje dorsal y ventral en las subespecies (Dunnum 2015). Rood (1972) menciona que las especies de Cavia tienen un pelaje dorsal agutí donde los pelos presentan una banda subterminal más clara y la punta es oscura. Adicionalmente, Eisenberg and Redford (1999) indican que en Perú los especímenes de $C$. tschudii son más oscuros.

En el Perú, Cavia tschudii se ubica en las ecorregiones de Puna, Serranía y Costa, teniendo como hábitat principal en la zona costera a los humedales (Pacheco et al. 2009). La especie ha sido registrada en el humedal RAMSAR los Pantanos de Villa, de manera casual al encontrarse un individuo muerto y una carcasa en un estudio de mamíferos (Pacheco et al. 2015). Continuando con los estudios en este humedal, en el presente trabajo reportamos el primer registro de albinismo del cuy silvestre Cavia tschudii.

\section{Material y métodos}

El espécimen albino fue capturado en el humedal costero los Pantanos de Villa (12¹2'48"S 7659'19"W), el cual se ubica en los límites de la zona urbana de Lima-Perú, en una depresión frente al Océano Pacífico, rodeado de colinas que alcanzan los 100 y 300 m de altitud (Instituto Nacional de Recursos Naturales -INRENA- 1998). Este humedal es considerado un refugio de aves migra- torias y es reconocido como un humedal de importancia internacional o sitio RAMSAR. A nivel nacional ha sido categorizado como Refugio de Vida Silvestre Los Pantanos de Villa (Decreto Supremo № 055-2006-AG) y es el humedal más importante de la costa central del Perú por su biodiversidad y hábitats (Cano \& Young 1998, Iannacone \& Alvariño 2007, Ramírez \& Cano 2010, Alarcón \& Iannacone 2014, Pacheco et al. 2015). El ejemplar albino fue capturado mediante una trampa Tomahawk, no se necesitó algún esfuerzo o diseño en la metodología de campo, adicional al estudio de la población de Cavia tschudii que se realizaba en el lugar.

\section{Resultados}

Capturamos una hembra albina adulta de Cavia tschudii en el humedal los Pantanos de Villa en agosto del 2018. Este ejemplar presentó las siguientes características: los ojos rojos; la piel expuesta o con pelaje corto en las patas y orejas, y la punta del hocico de color rosado; las garras trasparentes; el rostro, lados del cuerpo y dorso conformado con un pelaje crema en su totalidad. Los pelos son blancos con una banda color crema en la zona subterminal (Fig. 1). El fenotipo de este especimen corresponde al denominado albinismo platinum descrito por Beerman et al. (2004) en el ratón de laboratorio (Mus musculus), el cual se caracteriza por tener el color de ojos rojos, piel blanca y un pelaje claro (no blanco). La hembra capturada se encontró en una comunidad vegetal denominada Juncal (especie dominante Schoenoplectus americanus "junco") propia de los humedales costeros (Ramirez \& Cano 2010).

Este ejemplar es el primer registro de albinismo para una especie silvestre de la familia Caviidae y el segundo registro de albinismo para roedores en el Perú (Romero et al. 2018). El ejemplar albino se encuentra depositado en la colección científica del Departamento de Mastozoología, Museo de Historia Natural, Universidad Nacional Mayor de San Marcos (Lima, Perú) para alguna referencia posterior que puedan ser parte de algún estudio.

\section{Discusión}

El patrón de coloración hallado (fenotipo crema) contrasta fuertemente con la coloración observada en los demás ejemplares capturados $(\mathrm{N}=22)$, los cuales son caracterizados por un pelaje jaspeado oscuro, la base de los pelos negruzca, patas negras y garras oscuras (Figura 1). Cabe mencionar, que esta coloración fue bastante uniforme en todos los demás individuos capturados, sin presentar alguna variación apreciable. El fenotipo del ejemplar hallado podría ser parecido con un caso de leucismo por el pelaje crema, pero la presencia de ojos rojos y piel rosada nos permiten distinguirlo como albino (Romero \& Tirira 2017). Hallazgos con este color de pelaje claro, ojos rojos y piel rosada han sido reportados como casos de albinismo en otras especies de roedores del neotrópico (Martinez-Coronel et al. 2013, Romero et al. 2018, Stumpp et al. 2018).

Los patrones de coloración normal en mamíferos desempeñan múltiples funciones, las cuales pueden ser agrupadas principalmente en tres: camuflaje, comunicación y termorregulación, siendo el camuflaje el com- 


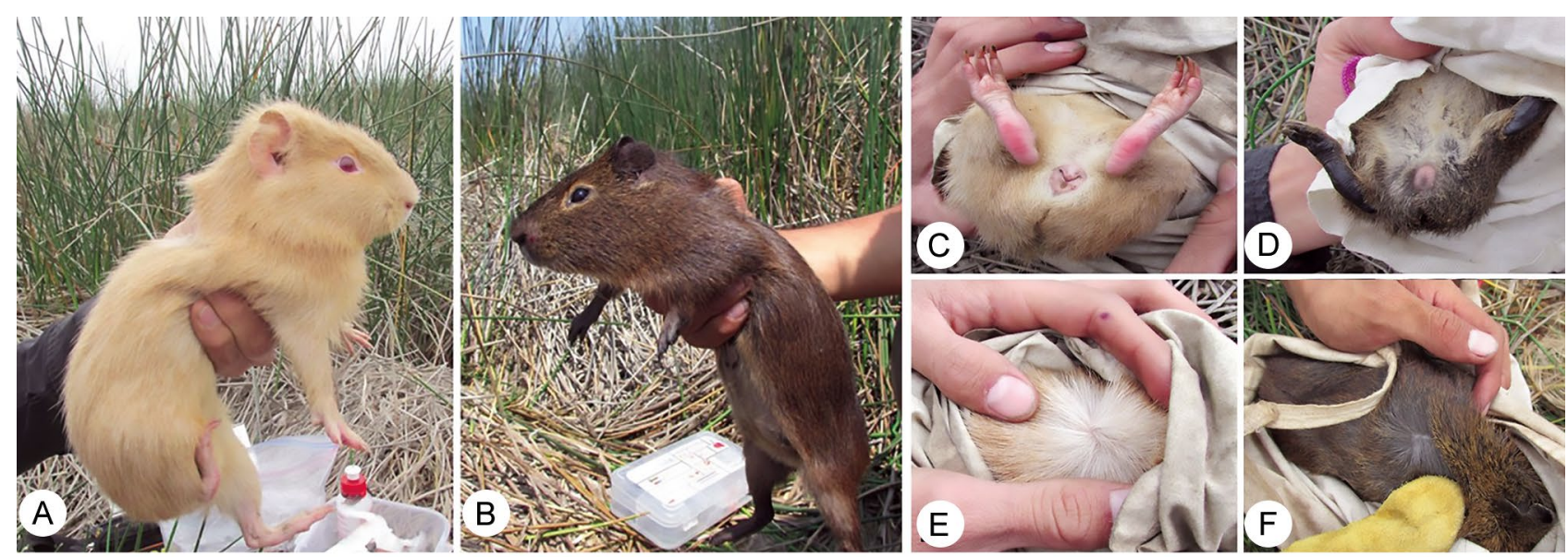

Figura 1. Comparación del ejemplar albino ( $\mathrm{A}, \mathrm{C}$ y E) y uno típico ( $\mathrm{B}, \mathrm{D}$ y F) del cuy silvestre (Cavia tschudii), ambos del humedal RAMSAR los Pantanos de Villa (Lima-Perú). En A y B se observan a los individuos recién capturados; C y D mostrando la coloración de las patas. E y $F$ mostrando la coloración del pelaje.

ponente más importante (Caro 2005). Por lo cual un organismo con una coloración diferente (albinismo) de lo normal puede estar sometido a una mayor presión de supervivencia en su ambiente natural (Vignieri et al. 2010). Bajo esta perspectiva los individuos albinos representarían una selección negativa para la especie debido a que su coloración los hace más visibles como depredadores o presas en su hábitat (Martinez-Coronel et al. 2013). Sin embargo, se ha reportado que organismos albinos de hábitos crípticos o nocturnos, como serpientes o peces, tienen mayor probabilidad de éxito que los albinos diurnos (Sazima \& Pombal 1986, Sazima \& Di Bernardo 1991). Esta afirmación se extiende para algunos roedores nocturnos (Stumpp et al. 2018) y se podría aplicar para $C$. tschudii por ser una especie de actividad nocturna.

El género Cavia es originario de Sudamérica y los reportes de mutaciones de color solo se habían realizado para la especie doméstica $C$. porcellus "guinea pigs" (Dunn 1921, Robinson 1973, Romero et al. 2018). Este hallazgo representa el primer reporte de albinismo para una especie silvestre ( $C$. tschudii) del género. Así mismo, el albinismo registrado podría ser un indicador indirecto de un proceso de endogamia a nivel poblacional en el área de estudio, ya que el humedal evaluado (263 ha) se encuentra rodeado de una matriz urbana (Lima) y no presenta una conexión física con otro ecosistema similar. Estas características del habitát podría estar generando una población pequeña y aislada cuya transferencia de genes entre parientes sería alta.

Los casos de albinismo han sido reportados con mayor frecuencia en poblaciones pequeñas y cerradas (Bensch et al. 2000, Chętnicki et al. 2007, Lopucki \& Mróz 2011) en dónde la endogamia es una de las causas del albinismo (Jannett 1981, Prado-Martinez et al. 2013) aumentando la probabilidad que se expresen genes recesivos que causan este tipo de anomalías. En este contexto, el albinismo reportado es importante, considerando las características particulares halladas para esta especie y el área de estudio; una población cerrada en un ecosistema insular rodeado de una matriz urbana.

\section{Literatura citada}

Acevedo J., D. Torres \& A. Aguayo-Lobo. 2009. Rare piebald and partially leucistic Antarctic fur seals, Arctocephalus gazella, at Cape Shirreff, Livingston Island, Antarctica. Polar Biology 32(1):41-45. DOI: https://doi. org/10.1007/s00300-008-0500-6

Acevedo J. \& M. Aguayo. 2008. Leucistic South American Sea Lion in Chile, with a review of anomalously color on otariids. Revista de Biología Marina y Oceanografía 43(2):413-417. DOI: http://dx.doi.org/10.4067/ S0718-19572008000200017

Alarcón G. \& J. Iannacone. 2014. Artropofauna terrestre asociada a formaciones vegetales en El Refugio de Vida Silvestre Pantanos de Villa, Lima, Perú. The Biologist 12(2):253-274.

Beerman F., S.J. Orlow \& M.L. Lamoreux. 2004. The Tyr (albino) locus of the laboratory mouse. Mammalian Genome 15(10):749-758. DOI: https://doi.org/10.1007/ s00335-004-4002-8

Bensch S., B. Hansson, D. Hasselquist \& B. Nielsen. 2000. Partial albinism in a semi-isolated population of great reed warblers. Hereditas 133(2):167-170. DOI: https:// doi.org/10.1111/j.1601-5223.2000.t01-1-00167.x

Blaszczyk W., C. Distler, G. Dekomien, L. Arning, K. Hoffmann \& J. Epplen. 2007. Identification of a tyrosinase (TYR) exon 4 deletion in albino ferrets (Mustela putorius furo). Animal Genetics 38(4):421-423. DOI: https:// doi.org/10.1111/j.1365-2052.2007.01619.x

Bui B.V. \& A.J. Vingrys. 1999. Development of receptoral responses in pigmented and albino guinea-pigs (Cavia porcellus). Documenta Ophthalmologica 99:151-170. DOI: https://doi.org/10.1023/a:1002721315955

Cano A. \& K.R. Young (Eds.). 1998. Los Pantanos de Villa: Biología y Conservación. Museo de Historia Natural, Universidad Nacional Mayor de San Marcos, Serie de Divulgación № 11. Lima, Perú.

Caro T. 2005. The adaptive significance of coloration in mammals. BioScience 55(2):125-136. DOI: https://doi. org/10.1641/0006-3568(2005)055[0125:TASOCI]2. $0 . \mathrm{CO} ; 2$

Chętnicki W., S. Fedyk \& U. Bajkowska. 2007. Cases of coat colour anomalies in the common shrew, Sorex araneus $\mathrm{L}$. Folia biológica 55(1-2):73-76. 
Cieslak M., M. Reissmann, M. Hofreiter \& A. Ludwig. 2011. Colours of domestication. Biological reviews of the Cambridge Philosophical Society 86(4):885-899. DOI: https://doi.org/10.1111/j.1469-185X.2011.00177.x

Dunn L. 1921. Unit character variation in rodents. Journal of Mammalogy 2(3):125-140. DOI: https://doi. org $/ 10.2307 / 1373601$

Dunnum J.L. 2015. Family Caviidae G. Fischer, 1817. Pp. 690726 in Mammals of South America (Patton, J. L., U. F. J. Pardiñas y G. D'Elía, eds). Volume 2: Rodents. The University of Chicago Press, Chicago, EE.UU.

Eisenberg J.F. \& Redford, K. H. 1999. Mammals of the Neotropics: Volume 3. The University of Chicaco Press, EE. UU.

Iannacone J. \& L. Alvariño. 2007. Diversidad y abundancia de comunidades zooplanctónicas litorales del humedal Pantanos de Villa, Lima, Perú. Gayana 71(1):49-65. DOI: http://dx.doi.org/10.4067/S071765382007000100006

Instituto Nacional de Recursos Naturales (INRENA). 1998. Plan Maestro de Los Pantanos de Villa. Ministerio de Agricultura. Lima, Perú.

Jannett F.J.Jr. 1981. Albinism and its inheritance in populations of the montane vole. Journal of Heredity 72(2):144146. DOI: https://doi.org/10.1093/oxfordjournals. jhered.a109453

Lacher T.E.Jr. 2016. Family Caviidae. Pp. 406 - 438 in Handbook of Mammals of the World (Wilson, D. E., T. E. Jr. Lacher, y R.A. Mittermeier, eds.). Vol. 6. Lagomorphs and Rodents: Part 1. Lynx Editions, Barcelona, Spain.

Lamoreux M., V. Delmas, L. Laure \& D. Bennett. 2010. The color of mice. A model genetic network. Wiley-Blackwell, Texas, EE.UU

Lopucki R. \& I. Mróz. 2011. Cases of colouration anomalies in small mammals of Poland, and reasons for their incidence. Annales Universitatis Mariae Curie-Sklodowska Lublin - Polonia 65(1): 67-76. DOI: https://doi. org/10.2478/v10067-011-0006-4

Martínez C., R. Bautista \& M. Verona. 2013. Albinismo platinado en Liomys pictus (Mammalia: Heteromyidae). Therya 4(3):641-645. DOI: http://dx.doi.org/10.12933/ therya-13-149

Pacheco V., A. Zevallos, K. Cervantes, J. Pacheco \& J. Salvador. 2015. Mamíferos del Refugio de Vida Silvestre Los Pantanos de Villa, Lima-Perú. Científica, 12: 26-41.

Peters R.H. 1983. The Ecological Implications of Body Size. Cambridge University Press, Cambridge, UK.

Prado-Martinez J., I. Hernando-Herraez, B. Lorente-Galdos, M. Dabad, O. Ramirez et al. 2013. The genome sequencing of an albino Western lowland gorilla reveals inbreeding in the wild. BMC Genomics 14:363. DOI: 10.1186/1471-2164-14-363

Ramirez D.W., H. Aponte, L. Gustavo \& F. Gil. 2018. Incendios en el humedal Ramsar Los Pantanos de Villa (Lima-Perú): Avances en su conocimiento y perspectivas futuras. Revista de Investigaciones Altoandinas 20(3):347360. DOI: http://dx.doi.org/10.18271/ria.2018.398.

Ramírez D. \& A. Cano. 2010. Estado de la diversidad de la flora vascular de los Pantanos de Villa (Lima-Perú). Revista Peruana de Biología 17(1):111-114. DOI: https://doi. org/10.15381/rpb.v17i1.58

Robinson R. 1973. Acromelanic albinism in mammals. Genetica 44(3):454-458. DOI: https://doi.org/10.1007/ BF00161317
Romero R., C. E. Rancines-Márquez \& J. Brito. 2018. A short review and worldwide list of wild albino rodents with the first report of albinism in Coendou rufescens (Rodentia: Erethizontidae). Mammalia, 82(5):509-515. DOI: https://doi.org/10.1515/mammalia-2017-0111

Romero V. \& D. Tirira. 2017. Leucistic Antarctic fur seal (Arctocephalus gazella) at Robert Island, South Shetland Islands, Antarctica, with a note on colour morph nomenclature. Polar Biology 40:1893-1897. DOI: https://doi.org/10.1007/s00300-016-2069-9

Rood J.P. 1972. Ecological and behavioural comparisons of three genera of Argentine cavies. Animal Behaviour Monographs, 5(1):3-83. DOI: https://doi.org/10.1016/ S0066-1856(72)80002-5

Stumpp R., D. Casali, H. Cunha \& A. Paglia. 2018. Complete albinism in Oxymycterus dasytrichus (Schinz 1821) (Rodentia: Cricetidae). Mammalia, 0(0), pp. -. Retrieved 19 Jan. 2019, from doi:10.1515/mammalia-2018-0005.

Treitler J.T., A. López Baucels, S. Gomes Farias, J. F. Tenaçol, Jr. \& R. Rocha. 2013. First record of a leucistic piebald Phyllostomus discolor (Chiroptera: Phyllostomidae). Chiroptera Neotropical 19(1):1178-1181.

Vignieri S.N., J.G. Larson \& H.E. Hoekstra. 2010. The selective advantage of crypsis in mice. Evolution 64(7):21532158. DOI: https://doi.org/10.1111/j.15585646.2010.00976.x

Wilson D., T. Lacher \& R. Mittermeier. 2017. Handbook of the Mammals of the World. Volumen 7. Rodents II. Lynx Editions. Barcelona, Spain.

Wright S. 1915. The albino series of allelomorphs in guineapigs. The American Naturalist 49:140-148.

\begin{abstract}
Agradecimientos:
El presente estudio se realizó con el apoyo financiero de la Dirección General de Investigación, Desarrollo e Innovación de la Universidad Científica del Sur, con quienes estamos muy agradecidos. También agradecemos al SERNANP y PROHVILLA por las autorizaciones brindadas para la presente investigación, así mismo quedamos profundamente agradecidos con los directores y guardaparques de cada institución quienes nos brindaron las facilidades logísticas durante el trabajo de campo.
\end{abstract}

Conflicto de intereses:

Los autores no incurren en conflictos de intereses.

Rol de los autores:

DWR escribió la primera versión del trabajo. MQ-L, DM-C, y VP, realizaron aportes y revisiones críticas al manuscrito.

Fuentes de financiamiento:

Dirección General de Investigación, Desarrollo e Innovación de la Universidad Científica del Sur.

\section{Aspectos éticos / legales:}

Autorización para realizar investigación en Áreas Naturales Protegidas del SINANPE: Resolución de la Jefatura del Refugio de Vida Silvestre Los Pantanos de Villa No. 008-2017-SERNANP-JEF. Certificado de Procedencia de Muestras Biológicas No. 04-2018-SERNANP-RVSLPV. 